\title{
Measuring Intrinsic Disorder and Tracking Conformational Transitions using Rosetta ResidueDisorder
}

Justin T. Seffernick, ${ }^{1}$ He Ren, ${ }^{2}$ Stephanie S. Kim, ${ }^{1}$ and Steffen Lindert ${ }^{1, *}$

${ }^{1}$ Department of Chemistry and Biochemistry, Ohio State University, Columbus, OH, 43210

${ }^{2}$ Department of Chemistry/Biochemistry, Oberlin College, Oberlin, OH, 44074

* Correspondence to:

Department of Chemistry and Biochemistry, Ohio State University

2114 Newman \& Wolfrom Laboratory, 100 W. $18^{\text {th }}$ Avenue, Columbus, OH 43210

614-292-8284 (office), 614-292-1685 (fax)

lindert.1@osu.edu

Table S1: Information on fast-folding protein dataset and long timescale MD simulations. ${ }^{1}$ Protein names correspond to sequences used in folding/unfolding trajectories. For four proteins (2f21, 1prb, 1mi0, and $1 \mathrm{lmb}$ ), which did not have either a crystal or NMR structure, a homologous sequence was used (bolded pdb codes) as the native structure. For the unfolding trajectories, the homologous sequence and structure from the pdb was used. $\mathrm{T}_{\mathrm{m}}$, $\mathrm{T}$, and Time (simulation time) for the long MD simulations were reported in Lindorff-Larsen et al. ${ }^{1}$

\begin{tabular}{|c|c|c|c|c|c|}
\hline Protein name & PDB code & Number of Residues & $\mathrm{T}_{\mathrm{m}}(\mathrm{K})$ & $\mathrm{T}(\mathrm{K})$ & Time $(\mu \mathrm{s})$ \\
\hline Chignolin & 2rvd & 10 & 381 & 340 & 106 \\
\hline Trp-cage & 2jof & 20 & 206 & 290 & 208 \\
\hline BBA & 1fme & 28 & - & 325 & 223 \\
\hline Villin & $2 \mathrm{f} 4 \mathrm{k}$ & 35 & 343 & 360 & 124 \\
\hline WW domain & $\mathbf{2 f 2 1}$ & 35 & 373 & 360 & 486 \\
\hline NTL9 & 2hba & 38 & 370 & 355 & 389 \\
\hline BBL & 2wxc & 47 & 251 & 298 & 272 \\
\hline Protein B & $\mathbf{1 p r b}$ & 47 & 217 & 340 & 104 \\
\hline Homeodomain & 2p6j & 52 & $>360$ & 360 & 198 \\
\hline Protein G & $\mathbf{1 m i 0}$ & 56 & 345 & 350 & 369 \\
\hline$\alpha 3 \mathrm{D}$ & $2 \mathrm{a} 2 \mathrm{~d}$ & 73 & 370 & 370 & 360 \\
\hline$\lambda$-repressor & $\mathbf{1 1 m b}$ & 80 & - & 350 & 161 \\
\hline
\end{tabular}


Table S2: RMSDs $(\AA)$ of selected structure of 12 fast-folding protein MD trajectories comparing cluster analysis performed by Lindorff-Larsen et al., clustering analysis performed by removing highly denatured frames using ResidueDisorder, and cluster analysis performed by removing high scoring structures using the Rosetta score.

\begin{tabular}{|c|c|c|c|}
\hline Protein & Lindorff-Larsen et al. $(\AA)$ & Rosetta ResidueDisorder $(\AA)$ & Rosetta Score $(\AA)$ \\
\hline $1 \mathrm{fme}$ & 1.6 & 2.8 & 2.8 \\
\hline $1 \mathrm{mmb}$ & 1.8 & 1.6 & 1.6 \\
\hline $1 \mathrm{mi} 0$ & 1.2 & 1.6 & 0.9 \\
\hline $1 \mathrm{prb}$ & 3.3 & 2.9 & 3.0 \\
\hline $2 \mathrm{a} 3 \mathrm{~d}$ & 3.1 & 2.9 & 2.9 \\
\hline $2 \mathrm{f} 21$ & 1.2 & 1.4 & 1.4 \\
\hline $2 \mathrm{f} 4 \mathrm{k}$ & 1.3 & 0.8 & 0.8 \\
\hline $2 \mathrm{hba}$ & 0.5 & 0.6 & 0.8 \\
\hline $2 \mathrm{jof}$ & 1.4 & 1.2 & 1.2 \\
\hline $2 \mathrm{p} 6 \mathrm{j}$ & 3.6 & 3.0 & 4.0 \\
\hline $2 \mathrm{rvd}$ & 1.0 & 1.2 & 1.3 \\
\hline $2 \mathrm{wxc}$ & 4.8 & 3.4 & 3.4 \\
\hline
\end{tabular}

Figure S1: SMD (10 - 25 ns simulations) percent denatured vs. time plots for the unfolding of six proteins (2rvd, 2joj, 2f4k, $2 \mathrm{wxc}, 2 \mathrm{p} 6 \mathrm{j}$, and $1 \mathrm{lmb}$ ). The color of the data points represents RMSD to the native structure (or closest homolog) for each frame. Figure 2 shows data for the remaining six proteins. As each protein unfolded, the percentage of denatured residues increased until the protein reached $100 \%$ denatured and constituted an extended chain.
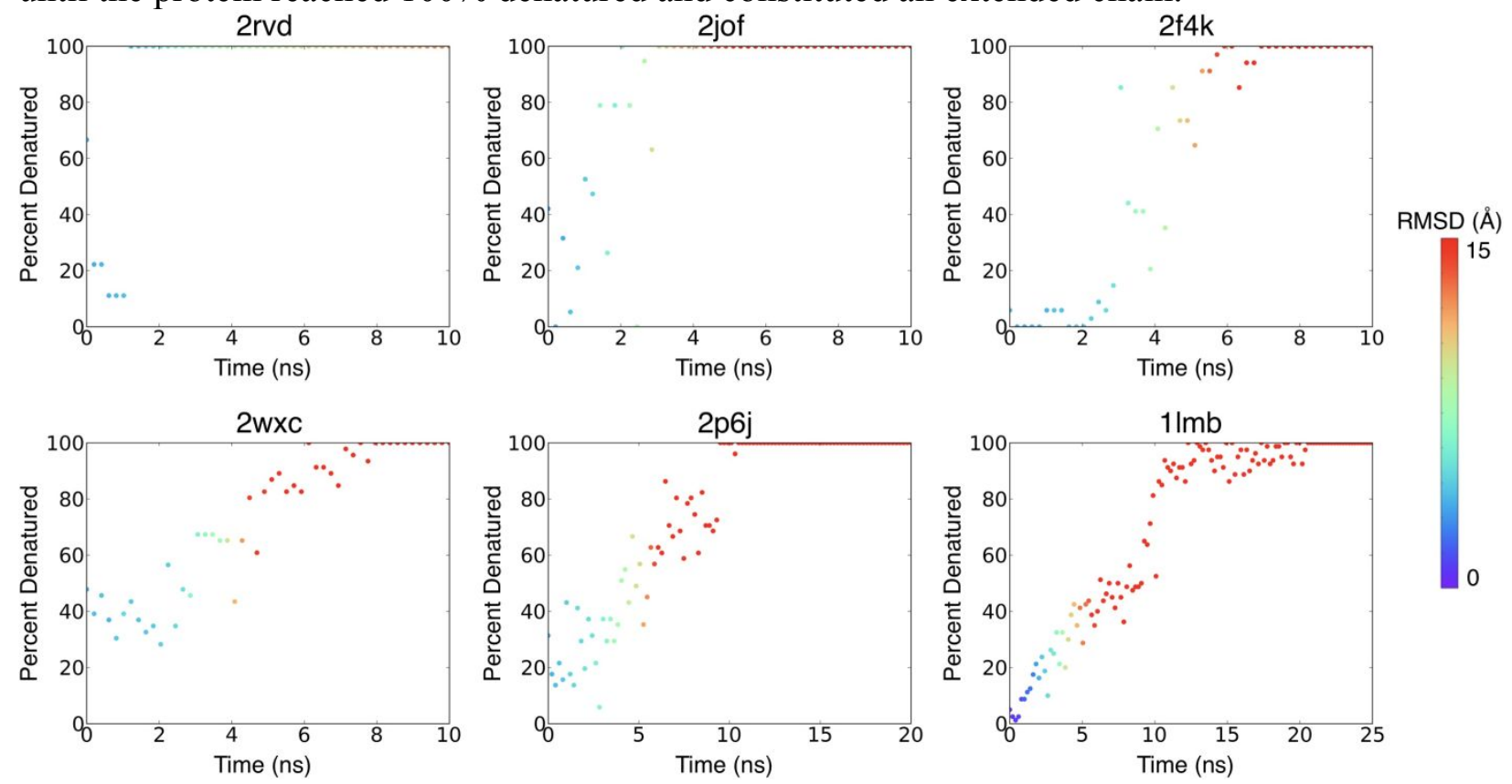
Figure S2: High temperature (500K) MD (200 ns simulations) percent denatured vs. time plots for the unfolding of six proteins (2rvd, $2 \mathrm{joj}, 2 \mathrm{f} 4 \mathrm{k}, 2 \mathrm{wxc}, 2 \mathrm{p} 6 \mathrm{j}$, and $1 \mathrm{lmb})$. The color of the data points represents RMSD to the native structure (or closest homolog) for each frame. Figure 3 shows data for the remaining six proteins.
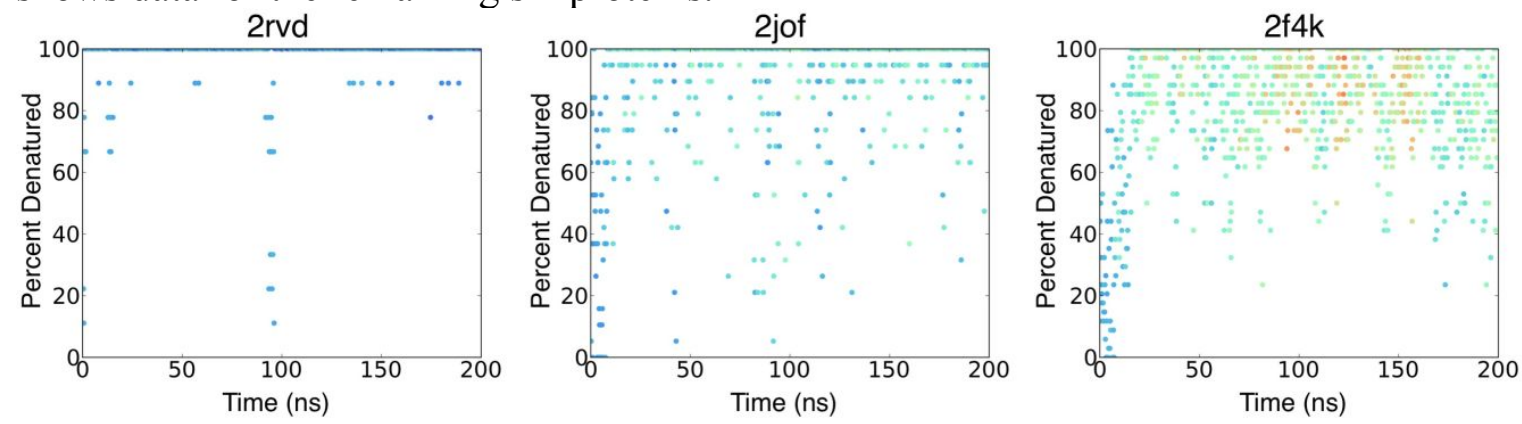

RMSD (Å)
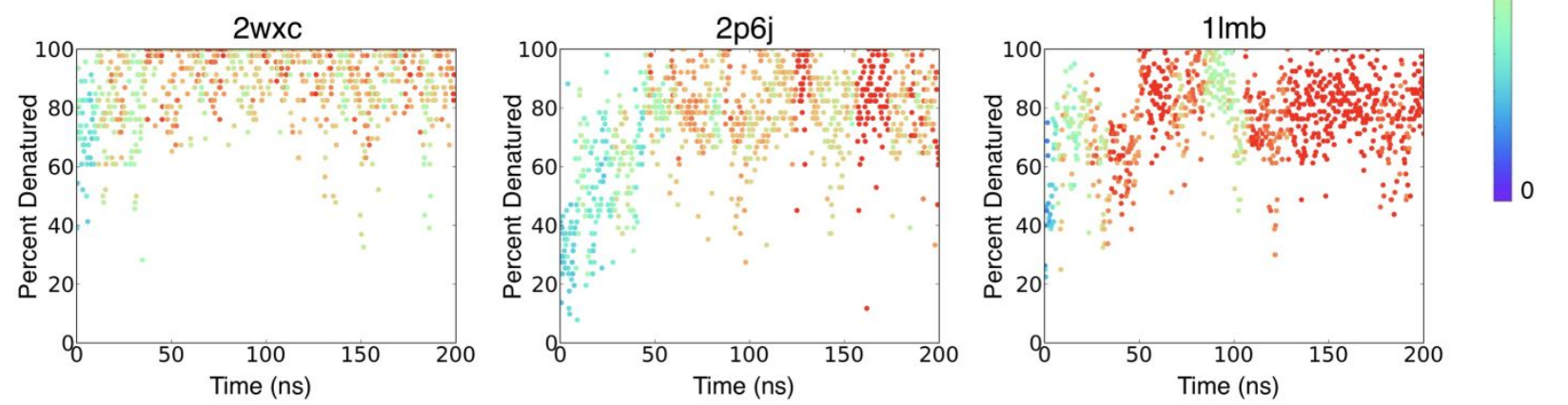
Figure S3: Accelerated MD (200 ns simulations) percent denatured vs. time plots for the unfolding of six proteins (2rvd, 2joj, 2f4k, 2wxc, 2p6j, and 1lmb). The color of the data points represents RMSD to the native structure (or closest homolog) for each frame. Figure 4 shows data for the remaining six proteins.
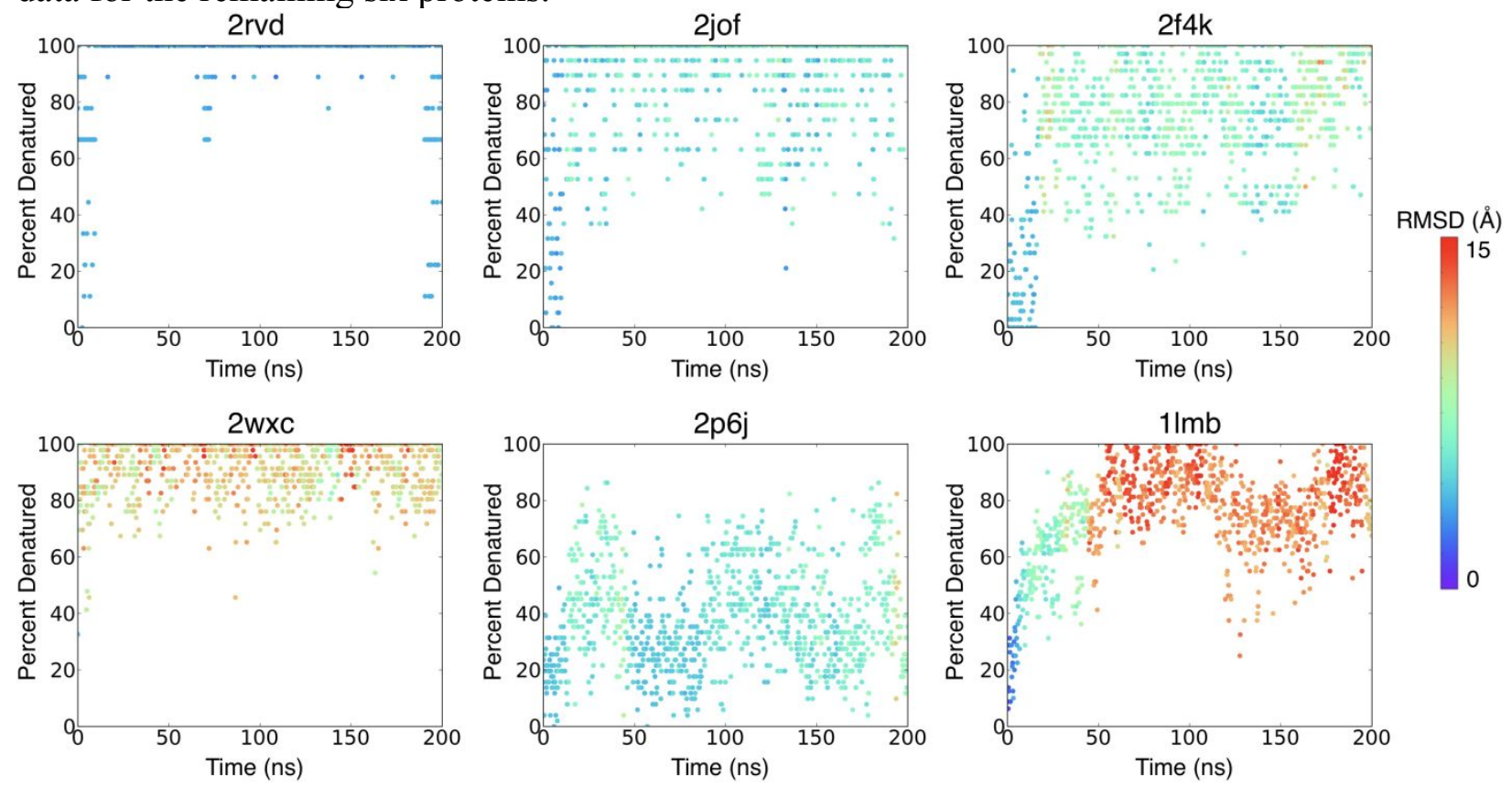
Figure S4: Percent denatured vs. time plots for the trajectories of six fast folding proteins (2rvd, $2 \mathrm{joj}, 2 \mathrm{f} 4 \mathrm{k}, 2 \mathrm{wxc}, 2 \mathrm{p} 6 \mathrm{j}$, and $1 \mathrm{lmb}$ ). Figure 5 shows data for the remaining six proteins. The color of the data points represents RMSD to the native structure (or closest homolog) for each frame. Rosetta ResidueDisorder separated the folded (low RMSD) states from the unfolded (high RMSD) states based on percentage of denatured residues. Highly non-denatured regions corresponded to low RMSD (folded) and highly denatured regions corresponded to high RMSD (unfolded).
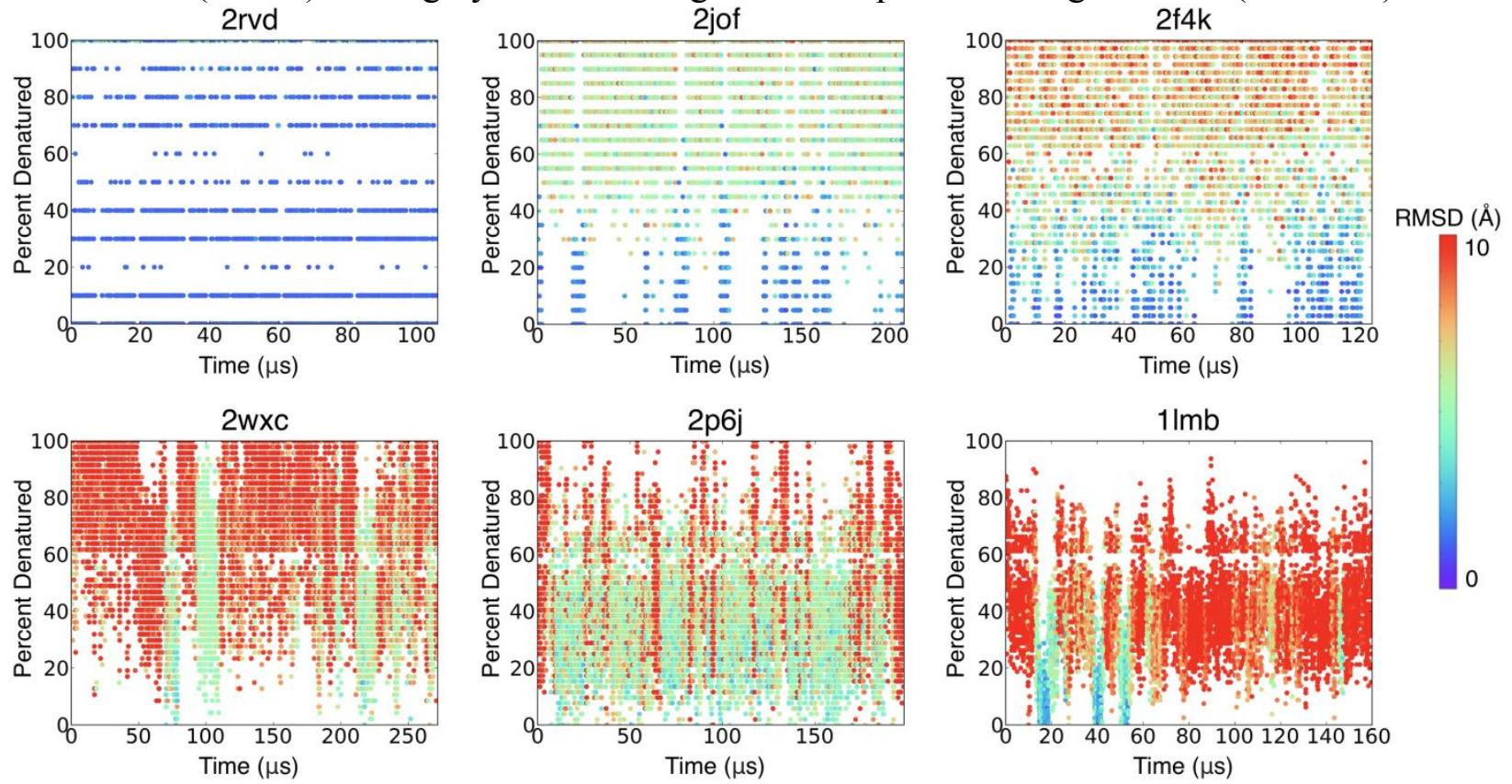
Figure S5: Percent denatured vs. RMSD plot over all frames for all 12 fast-folding proteins. In general, low RMSD structures correlated with highly non-denatured frames. Table 1 shows average RMSD over a range of denaturation, which increased in general.

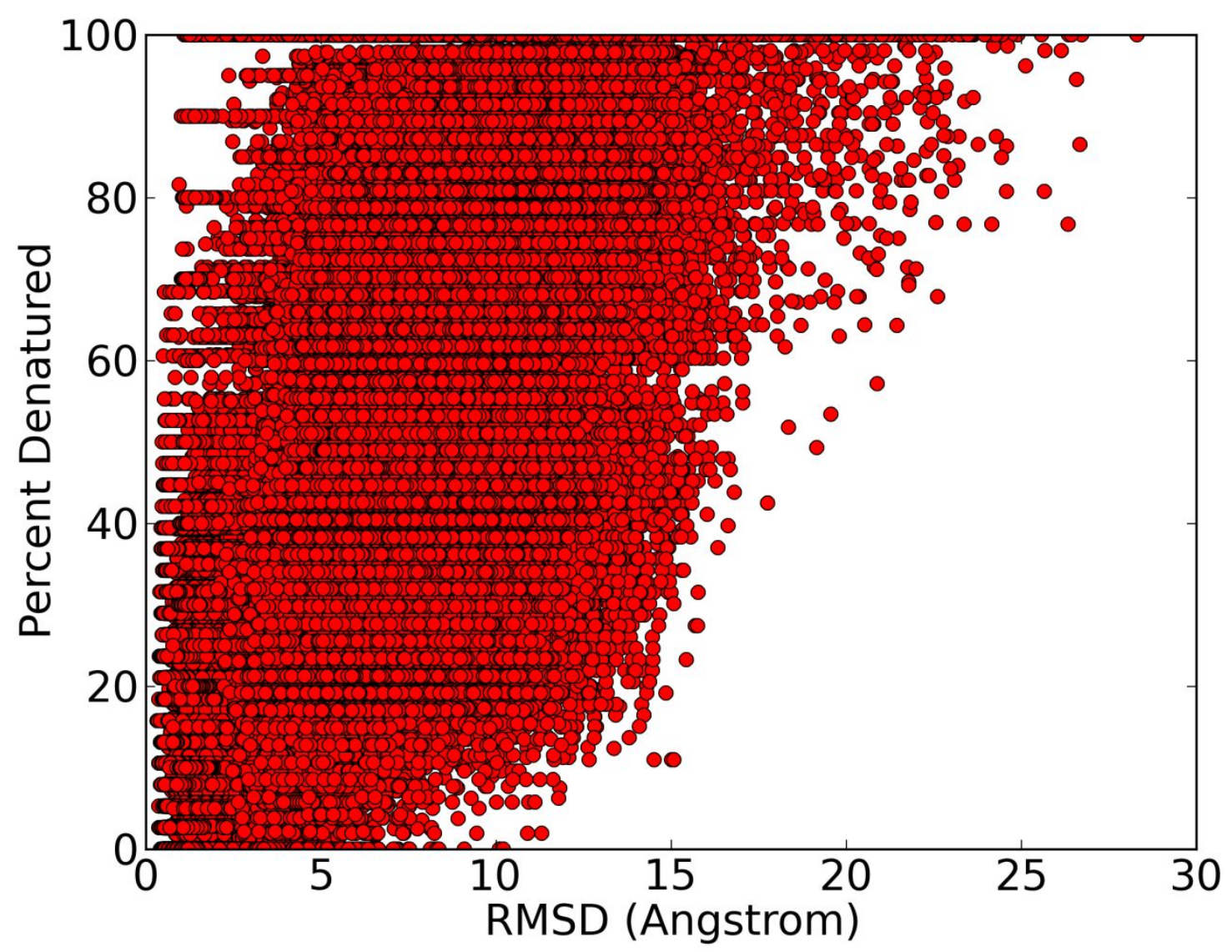


Figure S6: Percent denatured vs. time plots for six fast-folding proteins (2rvd, 2joj, 2f4k, $2 \mathrm{wxc}$, $2 \mathrm{p} 6 \mathrm{j}$, and $1 \mathrm{lmb}$ ) with ResidueDisorder predicted folding (solid) and unfolding (dotted) events shown as black vertical lines and TimeScapes terrain predicted events show as blue arrows for the fast folding protein trajectories. Figure 6 shows data for the remaining six proteins. RMSD to native for each frame is shown as a heatmap.
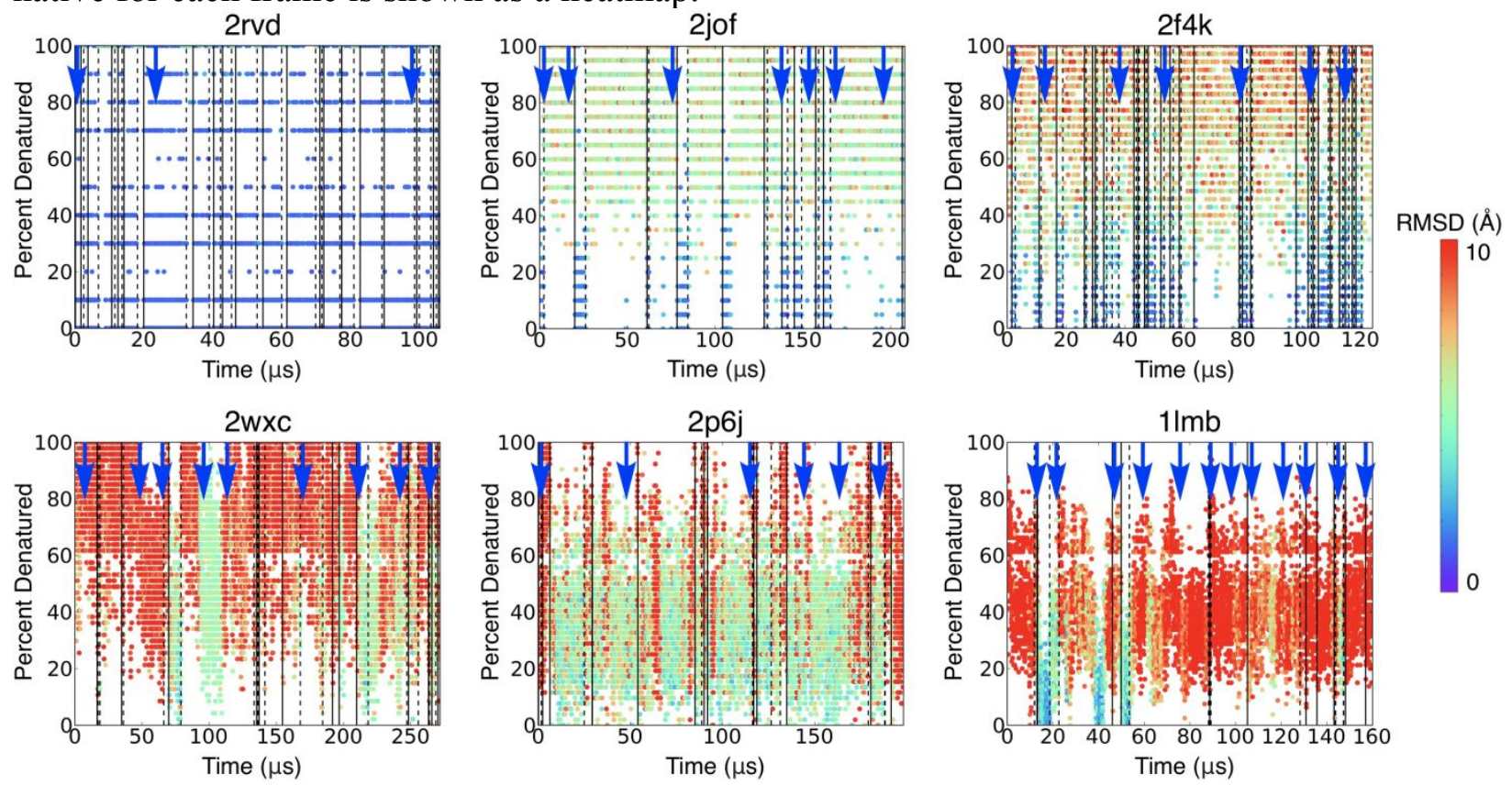
Figure S7: Comparison of folding (solid) and unfolding (dotted) events (shown as black, vertical lines) determined using the percentage of denatured residues from ResidueDisorder (left) and the total Rosetta score (right) for $1 \mathrm{fme}$ and $2 \mathrm{hba}$. The color of the data points represents RMSD to the native structure (or closest homolog) for each frame. While the overall accuracy (over all 12 proteins in the dataset) was comparable, for these two cases, the use of percent denatured more accurately predicted events than for when the Rosetta score was used.
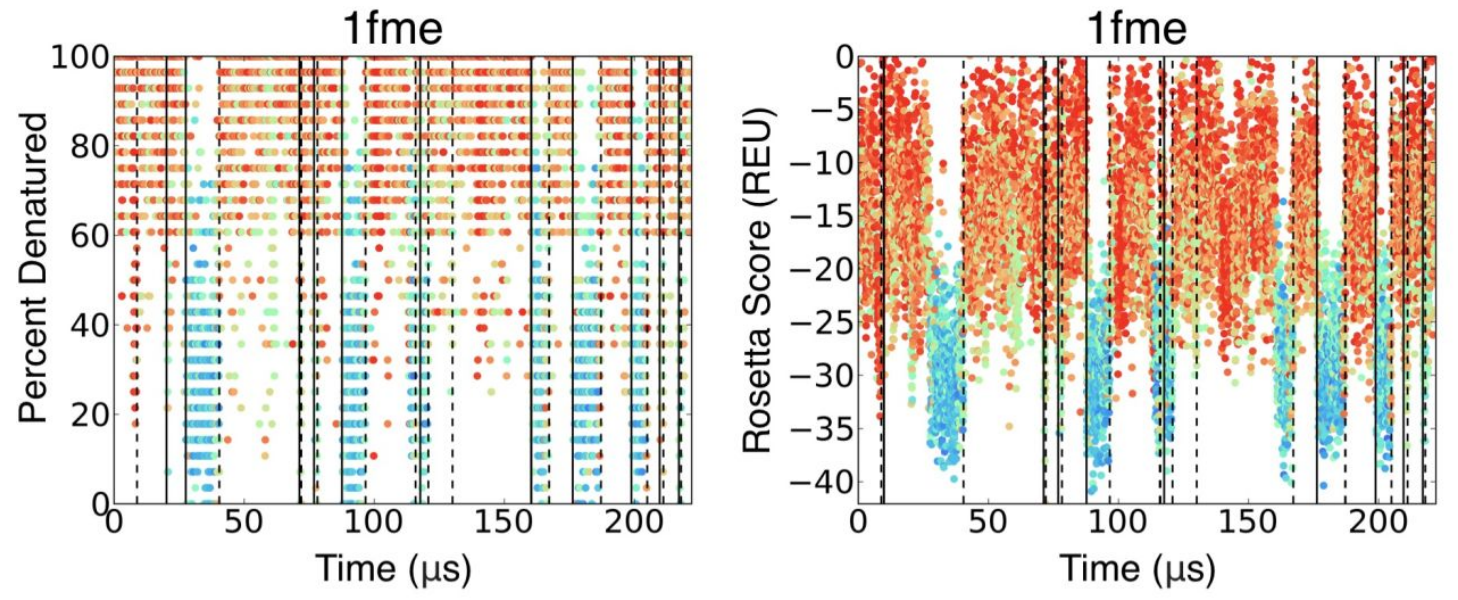

$\operatorname{RMSD}(\AA ̊)$
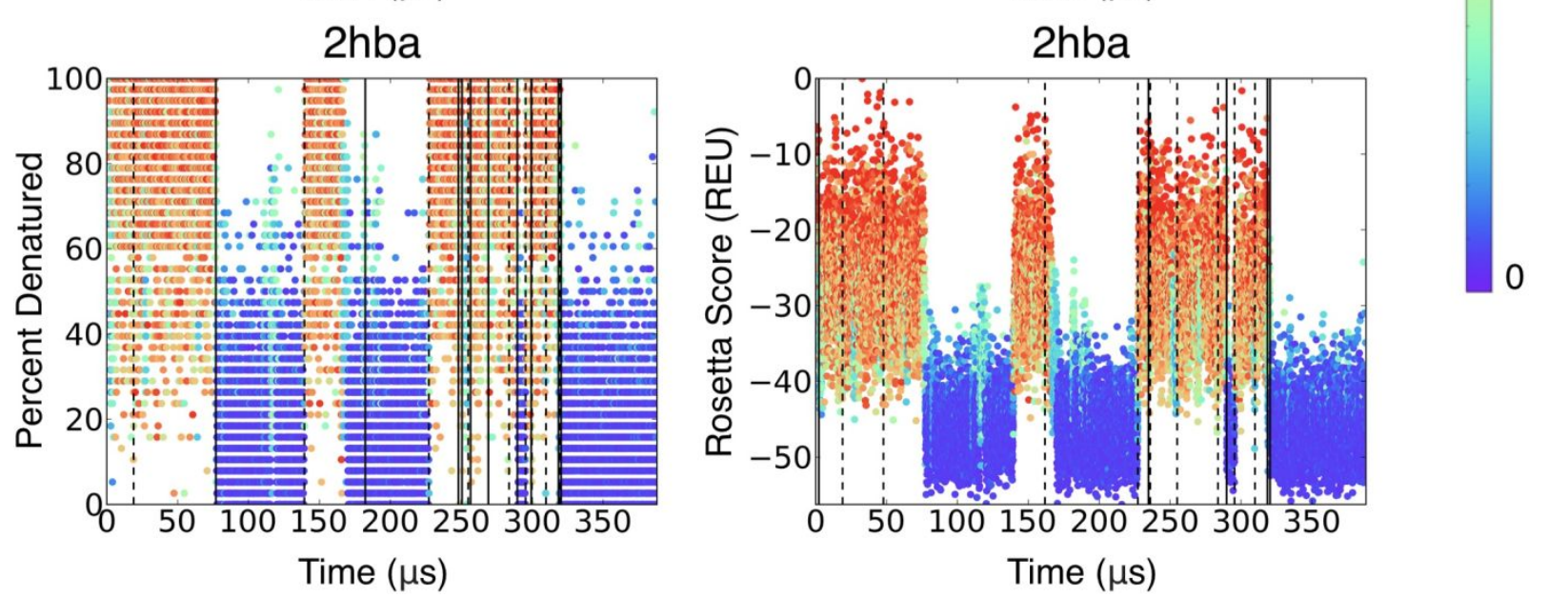


\section{ResidueDisorder application tutorial:}

There are three basic ways to run this Rosetta application:

1. Predict disorder from sequence

a. Instructions for this mode can be found as a tutorial in the Supporting Information of Kim et al. ${ }^{2}$ (Also see demo in $\sim$ Rosetta/demos/public/ResidueDisorder)

2. Measure disorder from structures

a. Before running the application, the structures must be relaxed (using the talaris2014 energy function). Relax documentation can be found at https:/www.rosettacommons.org/docs/latest/application_documentation/structure prediction/relax or run the following command

/Rosetta/relax.default. $\langle 0 s\rangle\langle$ compiler $\rangle$ release -database /Rosetta/main/database -in:file:l <input list text file> restore_talaris_behavior -out:prefix relax

b. Finally, run the ResidueDisorder application, inputting the relaxed structures. $\sim /$ Rosetta/ResidueDisorder. default. $\langle 0 s\rangle\langle$ compiler $\rangle$ release database $/$ Rosetta/main/database -in:file: l <input relax list text file>-restore_talaris_behavior measure_disorder_from_structure Optional: specify output file prefix with -out:prefix. This will output one file for each inputted structure.

3. Predict folding/unfolding events from structures

a. Before running the application, the structures must be relaxed (using the talaris2014 energy function). Repeat step 2a.

b. Finally, run the ResidueDisorder application, inputting the relaxed structures. Note: frames must be input in order. At least 50 frames must be input. /Rosetta/ResidueDisorder. default. $\langle 0 s\rangle\langle$ compiler $\rangle$ release database /Rosetta/main/database -in:file:l <input relax list text file>-restore_talaris_behavior measure_disorder_from_structure Optional: specify output file prefix with -out: prefix. This will output one file for each inputted structure. You can also specify the output file name for the list of folding/unfolding events with out:file: 0 . This will output one file listing the predicted events.

Documentation can be found at https://www.rosettacommons.org/docs/latest/ResidueDisorder. Demo can be found in $\sim$ Rosetta/demos/public/ResidueDisorder. Code can be found in $\sim$ Rosetta/main/source/src/apps/public/analysis/ResidueDisorder.cc. 


\section{References:}

1. Lindorff-Larsen, K.; Piana, S.; Dror, R. O.; Shaw, D. E., How Fast Folding Proteins Fold. Science 2011, 334, 517-520.

2. Kim, S. S.; Seffernick, J. T.; Lindert, S., Accurately Predicting Disordered Regions of Proteins Using Rosetta ResidueDisorder Application. J. Phys. Chem. B 2018, 122, 3920-3930. 